\title{
Artificial gauge fields for the Bose-Hubbard model on a checkerboard superlattice and extended Bose-Hubbard model
}

\author{
M. Iskin \\ Department of Physics, Koç University, Rumelifeneri Yolu, 34450 Sariyer, Istanbul, Turkey
}

(Dated: January 24, 2012)

\begin{abstract}
We study the effects of an artificial gauge field on the ground-state phases of the Bose-Hubbard model on a checkerboard superlattice in two dimensions, including the superfluid phase and the Mott and alternating Mott insulators. First, we discuss the single-particle Hofstadter problem, and show that the presence of a checkerboard superlattice gives rise to a magnetic flux-independent energy gap in the excitation spectrum. Then, we consider the many-particle problem, and derive an analytical mean-field expression for the superfluid-Mott and superfluid-alternating-Mott insulator phase transition boundaries. Finally, since the phase diagram of the BoseHubbard model on a checkerboard superlattice is in many ways similar to that of the extended Bose-Hubbard model, we comment on the effects of magnetic field on the latter model, and derive an analytical mean-field expression for the superfluid-insulator phase transition boundaries as well.
\end{abstract}

PACS numbers: 03.75.-b, 37.10.Jk, 67.85.-d

\section{INTRODUCTION}

In the usual Bose-Hubbard model [1], competition between the kinetic and potential energy terms leads to two phases: a Mott insulator (MI) when the kinetic energy is much smaller than the potential energy and a superfluid (SF) otherwise. The incompressible MI phase has an excitation gap so that the incoherent bosons are localized, and that a slight change in the chemical potential does not change the number of bosons on a particular lattice site. The compressible SF phase, however, is gapless, and the coherent bosons are delocalized over the entire lattice.

Recent advances with ultracold bosonic atoms loaded into optical lattices have made it possible to simulate BoseHubbard type many-particle Hamiltonians in a tunable setting. For instance, the ability to control on-site boson-boson interactions has paved the way for observing SF and MI phases as well as the transition between the two [1, 2]. In addition, a new technique has recently been developed that allowed the production of fictitious magnetic fields which can couple to neutral bosonic atoms [3, 4]. These fictitious magnetic fields are produced through an all optical Raman process, couple to a fictitious charge, but produce real effects like the creation of vortices in the SF state of bosons. Such an ability to control the strength of the fictitious magnetic fields combined with the ability to control the strength of the interparticle interactions may allow exploration of new phenomena in the near future [5-12].

In contrast to its simplicity, the Bose-Hubbard model is not exactly solvable even in one dimension. Therefore, it is desirable to have a much simpler toy-model which exhibits all the salient properties of the Bose-Hubbard model, while also being more amenable to analytical treatment. One of the most prominent candidates is the hardcore Bose-Hubbard model on a checkerboard superlattice, for which the existence of SF and MI phases at half filling in three dimensions [13], as well as a direct transition between the two [14] have rigorously been shown. In addition, this model and its correlation functions are exactly solvable in one dimension [15], thanks to the existence of a mapping between the hardcore bosons and noninteracting fermions. In the absence of a magnetic field, we have recently analyzed the ground-state phase diagram of this model in one, two and three dimensions using meanfield approximation and strong-coupling expansion, and compared them with the numerically exact results obtained from the stochastic series expansion algorithm followed by finitesize scaling [16]. Given that checkerboard superlattices have already been realized [19] using multiple wavelength laser beams, we extend previous works in two important directions. First, we relax the hardcore constraint and study the groundstate phase diagram of the softcore Bose-Hubbard model on a checkerboard superlattice, via mean-field decoupling approximation. Second, we study the effects of uniform magnetic field on the ground-state phase diagram in two dimensions.

The rest of this paper is organized as follows. First, we review the model at hand in Sec. III and present a qualitative description of its phase diagram. Then, we study the singleparticle Hofstadter problem in Sec. III] and show that the presence of a checkerboard superlattice gives rise to a magnetic flux-independent energy gap in the excitation spectrum. The many-particle problem is discussed in Sec. IV] where we derive analytical expressions for the SF-insulator phase transition boundaries within the mean-field decoupling approximation. Then, we give a brief discussion and summary of our results in Sec. V] Finally, we conclude the paper with Appendix A, where an analytical mean-field expression for the SF-insulator phase transition boundaries is derived for the extended Bose-Hubbard model.

\section{HAMILTONIAN}

In this paper we study the effects of magnetic field on the Bose-Hubbard model on a checkerboard superlattice. For this purpose, we consider a two-dimensional square lattice de- 
scribed by the Hamiltonian

$$
\begin{aligned}
H= & -\sum_{i j} t_{i j} e^{i \theta_{i j}} a_{i}^{\dagger} a_{j}+\frac{U}{2} \sum_{i} \widehat{n}_{i}\left(\widehat{n}_{i}-1\right) \\
& -C \sum_{i}(-1)^{\sigma_{i}} \widehat{n}_{i}-\mu \sum_{i} \widehat{n}_{i}
\end{aligned}
$$

where $a_{i}^{\dagger}\left(a_{i}\right)$ creates (annihilates) a boson on site $i$ and $\widehat{n}_{i}=a_{i}^{\dagger} a_{i}$ is the on-site boson number operator. The hopping matrix $t_{i j}$ is assumed to connect two nearest-neighbor lattice sites $\left(t_{i j}=t\right.$ for $i$ and $j$ nearest neighbors and 0 otherwise) belonging to different sublattices, e.g. the even sublattice $A$ and the odd one $B, U \geq 0$ is the strength of the on-site bosonboson repulsion, $\mu$ is the chemical potential, and $C \geq 0$ is the amplitude of the alternating checkerboard superlattice potential such that $\sigma_{i}=0(1)$ on sublattice $A(B)$. The phase factor $\theta_{i j}=\left(1 / \phi_{0}\right) \int_{i}^{j} \mathbf{A}_{\mathbf{0}}(\mathbf{r}) \cdot d \mathbf{r}$ takes into account the effects of a uniform magnetic field that is applied perpendicular to the lattice, where $\mathbf{A}_{\mathbf{0}}(\mathbf{r})$ is the vector potential and $\phi_{0}=h c / e$ is the magnetic flux quantum. All of our results recover the nonmagnetic ones when the magnetic flux tends to 0 .

Let us first analyze the atomic $(t=0)$ limit of this Hamiltonian at zero-temperature. In this limit, there is no kinetic term, and the boson number operator $\widehat{n}_{i}$ commutes with the Hamiltonian, so every lattice site is occupied by a fixed number $n_{i}=\left\langle\widehat{n}_{i}\right\rangle$ of bosons. Here, $\langle\ldots\rangle$ is the thermal average, and the average boson occupancy $n_{i}$ is chosen to minimize the ground-state energy for a given $\mu$. In particular, when $C=0$, this model is translationally invariant, and the groundstate boson occupancy is the same for all sites.

For instance, in the hardcore boson $(U \rightarrow \infty)$ limit [16], while the lattice is completely empty for $\mu<0$ and the minimal energy configuration corresponds to a vacuum of particles or a hole band insulator (VP, since $n_{i}=0$ for all $i$ ), it is completely full for $\mu>0$ and the minimal energy configuration corresponds to a vacuum of holes or a particle band insulator ( $\mathrm{VH}$, since $n_{i}=1$ for all $i$ ). The ground-state energy of these phases is degenerate at $\mu=0$. However, when $C>0$, the ground state has an additional half-filled insulating (incompressible) phase characterized by a crystalline order in the form of staggered boson densities. For the nearest neighbor lattice sites $i$ and $j,\left\langle\widehat{n}_{i}\right\rangle=n_{A}=1$ for the sublattice $A$ and $\left\langle\widehat{n}_{j}\right\rangle=n_{B}=0$ for the sublattice $B$. This phase resides in the region $|\mu|<C$, and it is sandwiched between the VP and VH phases. Since the checkerboard superlattice breaks the translational invariance of the lattice, it directly causes such an alternating density pattern. For this reason, this phase is often called a MI [14, 16], to distinguish it from a true chargedensity-wave (CDW) phase, for which the translational invariance is broken spontaneously due for instance to the presence of nearest-neighbor interactions. (See Appendix A). In this paper, for simplicity we call this alternating density pattern an alternating MI (AMI) with $(1,0)$ fillings to prevent confusion.

On one hand, for softcore bosons with $U>2 C \neq 0$, the ground state alternates between the AMI and MI phases as a function of increasing $\mu$, where the chemical potential widths of AMI and MI lobes are $2 C$ and $U-2 C$, respectively (see Fig. (4). On the other hand, for softcore bosons with $2 C>U$, the ground state has only AMI insulators. For instance, when $2 U>2 C>U$, the ground state is a VP with $(0,0)$ fillings for $\mu \leq-C$; it is an AMI with $(1,0)$ fillings for $\mu$ between $-C$ and $U-C$; it is an AMI with $(2,0)$ fillings for $\mu$ between $U-C$ and $C$; it is an AMI with $(2,1)$ fillings for $\mu$ between $C$ and $2 U-C$; it is an AMI with $(3,1)$ fillings for $\mu$ between $2 U-C$ and $U+C$, and so on. As $t$ increases, the range of $\mu$ about which the ground state is insulating decreases, and the MI and AMI phases disappear at a critical value of $t$, beyond which the system becomes compressible. We note that, unlike the compressible SF phase of the usual Bose-Hubbard model, the compressible phase in this model is more like a supersolid (SS), where the SF and AMI orders coexist even for arbitrarily small $C$. However, since the checkerboard superlattice breaks the translational invariance of the lattice, we call the compressible phase of this model a SF to distinguish it from a true SS for which the translational invariance is broken spontaneously. Having discussed the atomic limit, let us now discuss the noninteracting single-particle energy spectrum when $t \neq 0$.

\section{SINGLE-PARTICLE PROBLEM}

In the absence of a checkerboard superlattice, i.e. when $C=0$, the single-particle excitation spectrum is the usual Hofstadter butterfly [20], and here we generalize it to $C \neq 0$. For this purpose, we choose the Landau gauge for the vector potential $\left[\mathbf{A}_{\mathbf{0}}(\mathbf{r}) \equiv\left(0, B_{0} x, 0\right)\right]$, which leads to a uniform $\mathbf{B}_{\mathbf{0}}$ field in the $z$ direction, and the strength of the magnetic field $B_{0}$ is related to the magnetic flux $\Phi$ via $\Phi=B_{0} \ell^{2}$. Denoting the coordinates of lattice sites by $i \equiv(x=n \ell, y=m \ell)$, this gauge simply implies $\theta_{i j}=0$ for hoppings along the $x$ direction, i.e. between $(n, m)$ and $(n \pm 1, m)$; and $\theta_{i j}=$ $\pm 2 \pi \phi n$ for links along the $y$ direction, i.e. between $(n, m)$ and $(n, m \pm 1)$, where $\phi=\Phi /\left(2 \pi \phi_{0}\right)$. In the Landau gauge, the Hamiltonian given in Eq. (1) can be written as

$$
\begin{gathered}
H_{s p}=-t \sum_{n m}\left(a_{n m}^{\dagger} a_{n+1, m}+e^{i 2 \pi \phi n} a_{n m}^{\dagger} a_{n, m+1}+H . c .\right) \\
-C \sum_{n m}(-1)^{n+m} a_{n m}^{\dagger} a_{n m}
\end{gathered}
$$

where $n+m$ is even (odd) for sublattice $A(B)$. Here, we set $U=0$ and $\mu=0$ for the single-particle problem.

In the Landau gauge, taking $\phi=p / q$, where $p$ and $q$ are integers with no common factor, while the Hamiltonian given in Eq. (2) maintains its checkerboard translational invariance in the $y$ direction, i.e. it remains the same under 2 steps ( $m \rightarrow m+2$ ), it requires $q^{*}$ steps for translational invariance in the $x$ direction. For the square lattice considered, the period $q^{*}=q(2 q)$ for even (odd) $q$ values. Therefore, the Bloch theorem tells us that the 1 st magnetic Brillouin zone is determined by $-\pi / 2 \leq k_{y} \ell \leq \pi / 2$ and $-\pi / q^{*} \leq k_{x} \ell \leq \pi / q^{*}$. This increased periodicity motivates us to work with a supercell of $2 \times q^{*}$ sites (in $x$ and $y$ directions, respectively) as shown in Fig. 1 .

The single-particle excitation spectrum is determined by solving the Schrödinger equation 


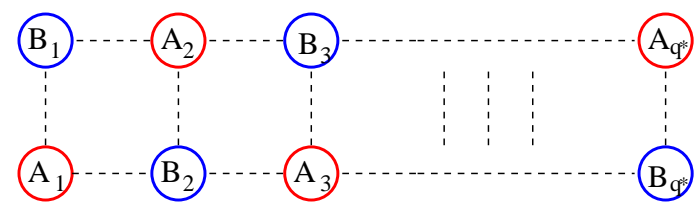

FIG. 1. (Color online) The $2 \times q^{*}$ supercell is shown where $q^{*}=q$ $(2 q)$ for even (odd) $q$ values.
$H_{s c} \Psi_{s c}=E_{C}(\phi) \Psi_{s c}$ for all $\mathbf{k}$ values in the $1 \mathrm{st}$ magnetic Brillouin zone. Choosing the wavefunction, $\Psi_{s c}=\left(\psi_{1}^{A}, \psi_{1}^{B}, \psi_{2}^{B}, \psi_{2}^{A}, \psi_{3}^{A}, \psi_{3}^{B}, \ldots, \psi_{q^{*}}^{B}, \psi_{q^{*}}^{A}\right)^{T}$, where $\psi_{n}^{A(B)}$ denotes the $n$th site of sublattice $A(B)$, the $2 q^{*} \times 2 q *$ matrix

$$
H_{s c}=\left[\begin{array}{cccccccccc}
-C & a_{1} & -t e^{-i k_{x} \ell} & 0 & 0 & 0 & . & 0 & -t e^{i k_{x} \ell} & 0 \\
a_{1} & +C & 0 & -t e^{-i k_{x} \ell} & 0 & 0 & . & 0 & 0 & -t e^{i k_{x} \ell} \\
-t e^{i k_{x} \ell} & 0 & +C & a_{2} & -t e^{-i k_{x} \ell} & 0 & . & 0 & 0 & 0 \\
0 & -t e^{i k_{x} \ell} & a_{2} & -C & 0 & -t e^{-i k_{x} \ell} & . & 0 & 0 & 0 \\
0 & 0 & -t e^{i k_{x} \ell} & 0 & -C & a_{3} & . & 0 & 0 & 0 \\
0 & 0 & 0 & -t e^{i k_{x} \ell} & a_{3} & +C & . & . & 0 & 0 \\
. & . & . & . & . & . & . & . & . & . \\
0 & 0 & 0 & 0 & 0 & . & . & . & . & -t e^{-i k_{x} \ell} \\
-t e^{-i k_{x} \ell} & 0 & 0 & 0 & 0 & 0 & . & . & +C & a_{q^{*}} \\
0 & -t e^{-i k_{x} \ell} & 0 & 0 & 0 & 0 & . & -t e^{i k_{x} \ell} & a_{q^{*}} & -C
\end{array}\right]
$$

describes the supercell with periodic boundary (Bloch) conditions. Here, $a_{n}=-2 t \cos \left(k_{y} \ell+2 \pi n p / q\right)$. Equation (3) is the generalization of the Hofstadter problem to the case of a checkerboard superlattice, and it reduces to the usual result when $C=0$ [20].

In Fig. 2, we show the single-particle excitation spectrum $E_{C}(\phi)$ as a function of the magnetic flux $\phi=p / q$ for two values of $C$ : (a) $C=0$ and (b) $C=t$. In both figures, $E_{C}(\phi)$ is shown to be symmetric around $p / q=1 / 2$, i.e. $E_{C}(\phi)=$ $E_{C}(1-\phi)$. This is simply because the magnetic flux values that add up to $2 \pi \phi_{0}$ are equivalent, e.g. $\phi=0$ and $\phi=1$. This also means that the maximal magnetic field $B_{0}$ that can be applied corresponds to $p / q=1 / 2$. A list of minimal singleparticle excitation energies $\epsilon(\phi)=\min _{\mathbf{k}} E_{0}(\phi) / t$ of the usual Hofstadter butterfly is also given for a number of $p / q$ values in Table. [.

In contrast to the presence of zero-energy excitations for all possible $\phi$ values in Fig. 2 (a), the most important difference in Fig. 2(b) is the presence of a flux-independent energy gap. Our numerical calculations show that the energy gap is exactly $2 C$. Since the on-site energy difference between the sublattice $\mathrm{A}$ and $\mathrm{B}$ is $2 \mathrm{C}$, this result is not so surprising at least in the $\phi \rightarrow 0$ limit. In an earlier work [16], in the absence of a magnetic field, we showed that the single-particle excitation spectrum becomes $E_{C}(0)= \pm \sqrt{C^{2}+E_{0}^{2}(0)}$, where $E_{0}(0)=-2 t \cos \left(k_{x} \ell\right)-2 t \cos \left(k_{y} \ell\right)$ is the usual singleparticle excitation spectrum. Similar to the nonmagnetic case, our numerical calculations show that

$$
E_{C}(\phi)= \pm \sqrt{C^{2}+E_{0}^{2}(\phi)}
$$

holds exactly in the presence of a magnetic field, where $E_{0}(\phi)=E_{C=0}(\phi)$ is the usual single-particle excitation spectrum of the Hofstadter butterfly. Having discussed the singleparticle problem, now we are ready to analyze the competition between the kinetic and potential energy terms of the manyparticle Hamiltonian when $t \neq 0$.

TABLE I. A list of minimal single-particle excitation energies $\epsilon(\phi)=\min _{\mathbf{k}} E_{0}(\phi) / t$ of the usual Hofstadter butterfly $(C=0)$ is given for a number of magnetic flux $\phi=\Phi /\left(2 \pi \phi_{0}\right)=p / q$ values.

\begin{tabular}{cc|cc|cc|cc}
\hline \hline$p / q$ & $\epsilon(\phi)$ & $p / q$ & $\epsilon(\phi)$ & $p / q$ & $\epsilon(\phi)$ & $p / q$ & $\epsilon(\phi)$ \\
\hline $1 / 2$ & -2.828 & $2 / 3$ & -2.732 & $3 / 4$ & -2.828 & $4 / 5$ & -2.966 \\
$1 / 3$ & -2.732 & $2 / 5$ & -2.618 & $3 / 5$ & -2.618 & $4 / 7$ & -2.611 \\
$1 / 4$ & -2.828 & $2 / 7$ & -2.725 & $3 / 7$ & -2.611 & $4 / 9$ & -2.630 \\
$1 / 5$ & -2.966 & $2 / 9$ & -2.881 & $3 / 8$ & -2.613 & $4 / 11$ & -2.626 \\
$1 / 6$ & -3.096 & $2 / 11$ & -3.028 & $3 / 10$ & -2.698 & $4 / 13$ & -2.692 \\
$1 / 7$ & -3.203 & $2 / 13$ & -3.151 & $3 / 11$ & -2.746 & $4 / 15$ & -2.761 \\
$1 / 8$ & -3.291 & $2 / 15$ & -3.249 & $3 / 13$ & -2.854 & $4 / 17$ & -2.842 \\
$1 / 9$ & -3.362 & $2 / 17$ & -3.328 & $3 / 14$ & -2.906 & $4 / 19$ & -2.920 \\
$1 / 10$ & -3.420 & $2 / 19$ & -3.392 & $3 / 16$ & -3.005 & $4 / 21$ & -2.994 \\
$1 / 11$ & -3.469 & $2 / 21$ & -3.445 & $3 / 17$ & -3.050 & $4 / 23$ & -3.061 \\
$1 / 12$ & -3.510 & $2 / 23$ & -3.490 & $3 / 19$ & -3.132 & $4 / 25$ & -3.123 \\
$1 / 13$ & -3.545 & $2 / 25$ & -3.528 & $3 / 20$ & -3.168 & $4 / 27$ & -3.177 \\
$1 / 14$ & -3.576 & $2 / 27$ & -3.561 & $3 / 22$ & -3.234 & $4 / 29$ & -3.226 \\
$1 / 15$ & -3.602 & $2 / 29$ & -3.590 & $3 / 23$ & -3.263 & $4 / 31$ & -3.263 \\
\hline \hline
\end{tabular}


( a)

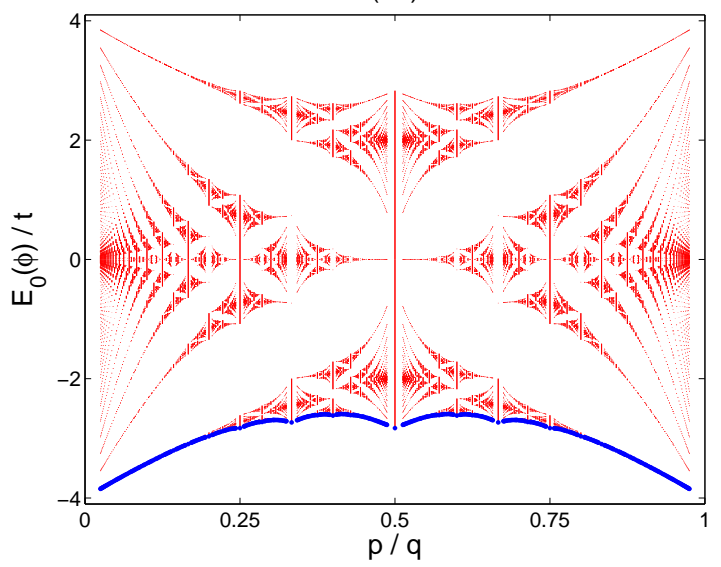

(b)

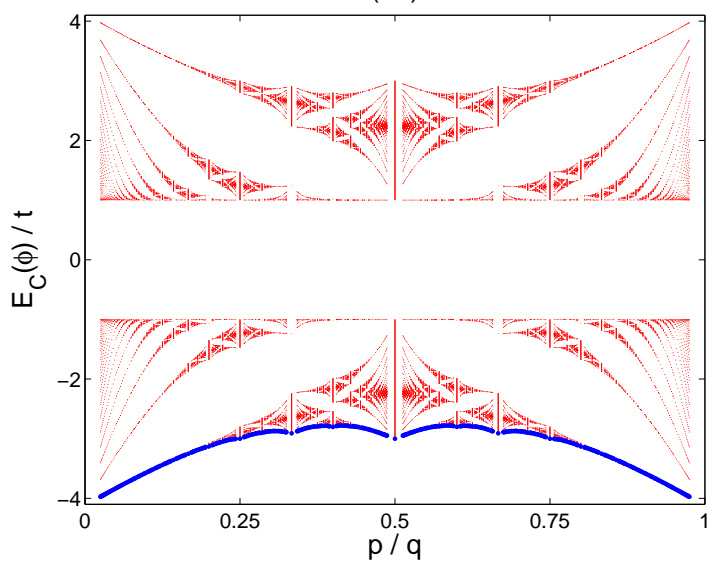

FIG. 2. (Color online) The single-particle excitation spectrum $E_{C}(\phi)$ of the Hamiltonian given in Eq. (3) (in units of $t$ ) is shown as a function of the magnetic flux $\phi=\Phi /\left(2 \pi \phi_{0}\right)=p / q$ for two values of $C$ : (a) $C=0$ and (b) $C=t$. Note that a flux-independent energy gap opens in (b), which exactly equals to $2 C$.

\section{MANY-PARTICLE PROBLEM}

For the many-particle problem, we add the boson-boson interaction and chemical potential terms to Eq. (2), and obtain

$$
\begin{aligned}
H_{m p}= & -t \sum_{n m}\left(a_{n m}^{\dagger} a_{n+1, m}+e^{i 2 \pi \phi n} a_{n m}^{\dagger} a_{n, m+1}+H . c .\right) \\
& +\frac{U}{2} \sum_{n m} a_{n m}^{\dagger} a_{n m}\left(a_{n m}^{\dagger} a_{n m}-1\right) \\
& -C \sum_{n m}(-1)^{n+m} a_{n m}^{\dagger} a_{n m}-\mu \sum_{n m} a_{n m}^{\dagger} a_{n m} .
\end{aligned}
$$

For illustrative purposes, let us first study the ground-state phase diagram of this model for the hardcore bosons, for which the calculation becomes considerably simpler compared to the softcore case, but yet nontrivial.

\section{A. Hardcore bosons}

The hardcore boson operators satisfy the constraint $a_{n m}^{\dagger 2}=$ $a_{n m}^{2}=0$, which prohibit multiple occupancy of lattice sites, as dictated by the infinitely large on-site boson-boson repulsion $(U \rightarrow \infty)$. In this limit, the many-particle Hamiltonian given in Eq. (5) becomes invariant under the transformation $a_{n m} \rightarrow a_{n \pm 1, m}^{\dagger}$ or $a_{n m} \rightarrow a_{n, m \pm 1}^{\dagger}$, which corresponds to a shift of one lattice site in $x$ or $y$ direction. This symmetry operation, which can be immediately read off from the Hamiltonian, corresponds to a particle-hole exchange combined with swapping $A$ and $B$ sublattices. It leads to a $\mu \rightarrow-\mu$ symmetry, i.e. the phase diagram is symmetric around $\mu=0$.

It turns out that the exact SF-VP and SF-VH phase transition boundaries can be easily obtained analytically. The simplest argument leading to this conclusion stems from the fact that this boundary is determined by the addition of a single particle (hole) to the completely-empty (-filled) lattice. It can then be argued that whether one is dealing with hardcore bosons or noninteracting spinless fermions makes no difference, as the particle statistics plays no role. This further means that one needs only to diagonalize the single-particle Hamiltonian and find the energy difference between the completelyempty (-filled) lattice and the state with one particle (hole). The single-particle spectrum is already given in Eq. (4), and this procedure leads to

$$
\mu= \pm \sqrt{C^{2}+\epsilon^{2}(\phi) t^{2}}
$$

where $\epsilon(\phi)=\min _{\mathbf{k}} E_{0}(\phi) / t$ are the minimal single-particle excitation energies of the usual Hofstadter butterfly which are shown as big blue dots in Fig. 2. a). In other words, the minus (plus) branch in Eq. (6) is determined by the minimal (maximal) single-particle excitation energies of the Hofsdtadter butterfly when $C \neq 0$. The minimal single-particle excitation energies are shown as big blue dots in Fig. 2(b). We emphasize that Eq. (6) is exact for two-dimensional square lattices, and the minus (plus) sign determines the SF-VP (-VH) phase transition boundary. Note again that Eq. (6) reduces to the known result for the nonmagnetic case [16] in the $\epsilon(\phi \rightarrow 0)=-4$ limit.

Unlike the SF-VP and SF-VH phase boundaries, the SFAMI phase transition boundary cannot be determined exactly, since the exact many-particle wave function for the AMI state is not known. However, this can be achieved via the mean-field decoupling approximation. Within this approximation, the hopping terms in the Hamiltonian given in Eq. (5) are decoupled according to, $a_{n m}^{\dagger} a_{n+1, m}=\left\langle a_{n m}^{\dagger}\right\rangle a_{n+1, m}+$ $a_{n m}^{\dagger}\left\langle a_{n+1, m}\right\rangle-\left\langle a_{n m}^{\dagger}\right\rangle\left\langle a_{n+1, m}\right\rangle$, where the expectation values $\varphi_{n m}(\phi)=\left\langle a_{n m}\right\rangle$ correspond to the mean-field SF order parameters. Note that there are at most $2 q^{*}$, i.e. total number of sites in a supercell, distinct $\varphi_{n m}$ and the SF (AMI) phase is determined by the nonzero (zero) value of any one (all) of them. In particular, there are two distinct SF order parameters (one for each sublattice) in the nonmagnetic case even for arbitrarily small $C$, as long as $C \neq 0$. We checked that these order parameters differ from each other for all parameter space via Gutzwiller ansatz calculations. Therefore, unlike 
the compressible SF phase of the usual Bose-Hubbard model, the SF phase in the checkerboard model is more like a SS, where the SF and AMI orders coexist.

Performing a second-order perturbation theory in $\varphi_{n m}$ around the MI phase, and following the usual Landau procedure for second-order phase transitions, i.e. minimizing the ground-state energy as a function of $\varphi_{n m}$, we eventually arrive at the phase transition boundary equation

$$
\mu= \pm \sqrt{C^{2}-\epsilon^{2}(\phi) t^{2}}
$$

where the plus (minus) sign corresponds to particle (hole) excitations above the AMI phase. We note that although $\varphi_{n m}$ are gauge dependent, the phase transition boundary itself is not [6, 7, 9]. Alternatively, Eq. (7) follows directly from the strong-coupling expansion of the ground-state energy of the AMI phase with respect to the hopping term [16], where the first nontrivial hopping dependence of the phase transition boundary arises from the maximal eigenvalue of the $\mathbf{T}=\mathbf{t} \cdot \mathbf{t}$ matrix [21]. Here, the elements of $T_{i j}=\sum_{k} t_{i k} t_{k j}$ are such that $\sum_{j} T_{i j} f_{j}=\epsilon^{2}(\phi) t^{2} f_{i}$. Note again that Eq. (7) reduces to the known result for the nonmagnetic case [16] in the $\epsilon(\phi \rightarrow 0)=-4$ limit.

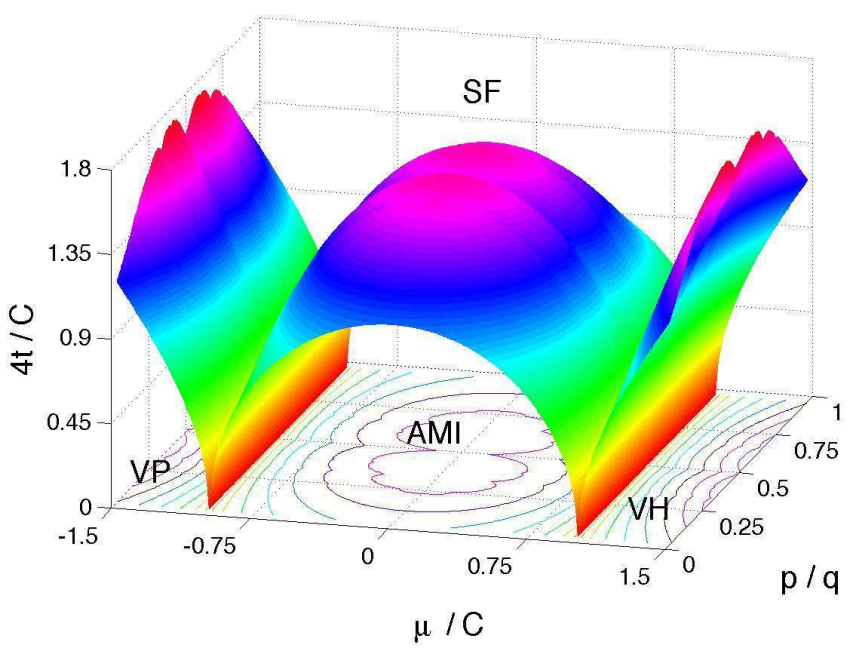

FIG. 3. (Color online) The ground-state phase diagram of hardcore bosons is shown as a function of the chemical potential $\mu$ (in units of $C$ ), hopping $4 t$ (in units of $C$ ) and magnetic flux $\phi=\Phi /\left(2 \pi \phi_{0}\right)=$ $p / q$, as obtained from Eqs. (6) and (7). The phase diagram is symmetric around $\mu=0$ and $p / q=1 / 2$ as explained in the text, and the intriguing structure of the phase boundaries (which is clearly seen in the contour plot) is due to the minimal single-particle excitation energies $\epsilon(\phi)$ shown as big blue dots in Fig. 2]a).

In Fig. 3, we show the ground-state phase diagram as a function of the chemical potential $\mu$, hopping $4 t$ and magnetic flux $\phi=p / q$, that is obtained from Eqs. (6) and (7). As we argued above, the phase diagram is symmetric around $\mu=0$ and $p / q=1 / 2$. The latter symmetry is in agreement with the earlier findings on the usual Bose-Hubbard model [57]. What is more interesting is the intriguing structure of the phase transition boundaries on the minimal single-particle ex- citation energies $\epsilon(\phi)$. In addition, the incompressible (compressible) AMI (SF) phase grows (shrinks) when the magnetic field increases from zero, due to the localizing effects of the magnetic field on bosons. All of these observations are similar to earlier findings on the usual Bose-Hubbard model [5-7].

\section{B. Softcore bosons}

Having studied the effects of magnetic field on the hardcore bosons, let us now analyze the ground-state phase diagram of the Hamiltonian given in Eq. (5) for the softcore bosons. Since the exact many-particle wave functions for the AMI and MI states are not known, we again obtain the phase diagram via the mean-field decoupling approximation. Following the recipe given in the previous section, the SF-MI and SF-AMI phase transition boundaries are found to be determined by

$$
\begin{gathered}
\frac{1}{\epsilon^{2}(\phi) t^{2}}=\left[\frac{n_{A}+1}{U n_{A}-C-\mu}-\frac{n_{A}}{U\left(n_{A}-1\right)-C-\mu}\right] \\
\times\left[\frac{n_{B}+1}{U n_{B}+C-\mu}-\frac{n_{B}}{U\left(n_{B}-1\right)+C-\mu}\right],
\end{gathered}
$$

which gives a quartic equation for $\mu$. This equation is valid for all $C$, and $\epsilon(\phi)=\min _{\mathbf{k}} E_{0}(\phi) / t$ are the minimal singleparticle excitation energies of the usual Hofstadter butterfly. In the hardcore $(U \rightarrow \infty)$ limit, note that Eq. (8) reduces to Eq. (7) when $n_{A}=1$ and $n_{B}=0$, and to Eq. (6) when $n_{A}=n_{B}=0$ or $n_{A}=n_{B}=1$. In addition, Eq. (8) reduces to the known result [7] for the usual Bose-Hubbard model when $n_{A}=n_{B}=n_{0}$ and $C=0$, and it also agrees with the recent numerical calculations [17, 18] in the absence of a magnetic field. Since a simple closed form analytic solution for $\mu$ is not possible when $C \neq 0$, we solve Eq. (8) with MATHEMATICA for each of the AMI and MI lobes separately.

In Fig. 4, we set $C=0.2 U$ and show the ground-state phase diagram as a function of the chemical potential $\mu$ and hopping $4 t$ for $p / q=1 / 1$ (equivalent to zero magnetic field) and for $p / q=1 / 2$ (maximum magnetic field). As discussed in Sec. [II the ground state alternates between the AMI and MI phases as a function of increasing $\mu$. The chemical potential widths of AMI and MI lobes are $0.4 U$ and $0.6 U$, respectively, but the size of the AMI (MI) lobes increase (decrease) as a function of increasing $C / U$ (not shown), since a nonzero $C$ is what allowed AMI states to form in the first place. In addition, the incompressible (compressible) AMI and MI (SF) phases grow (shrinks) when the magnetic field increases from zero, due again to the localizing effects of the magnetic field on bosons. Since the phase diagram of the Bose-Hubbard model on a checkerboard superlattice is in many ways similar to that of the extended Bose-Hubbard model, we discussed the latter model in Appendix A.

\section{CONCLUSIONS}

In this paper, we studied ground-state phases of the BoseHubbard model on a checkerboard superlattice in two dimen- 


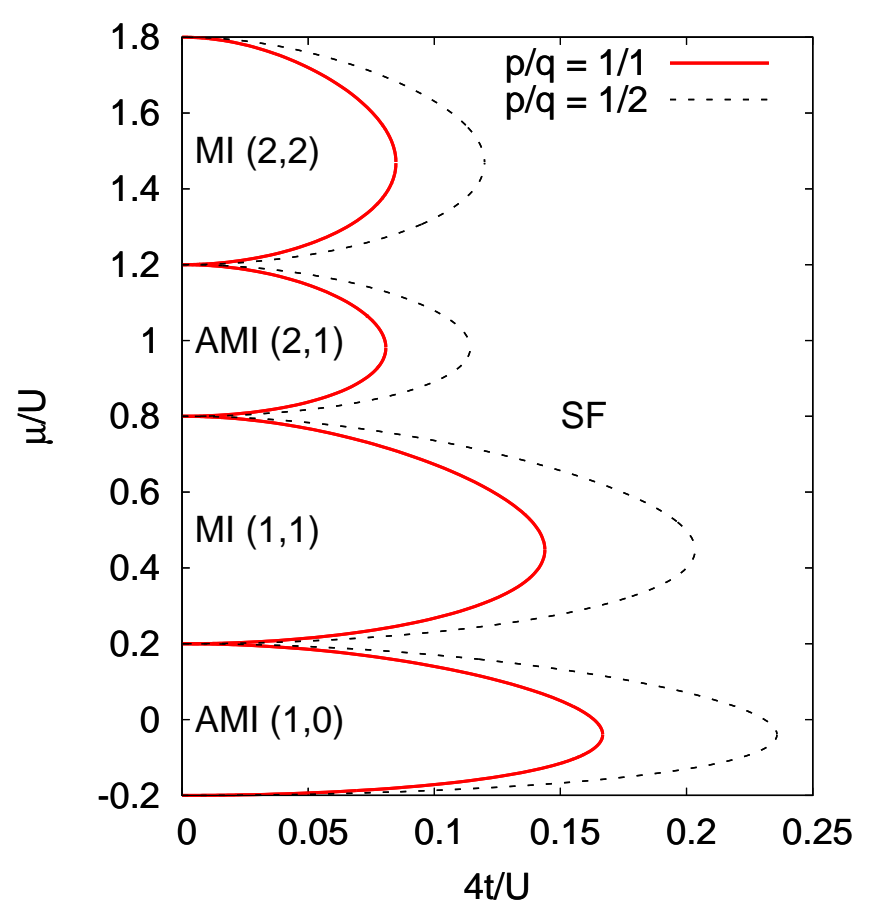

FIG. 4. (Color online) The ground-state phase diagram of softcore bosons is shown as a function of the chemical potential $\mu$ (in units of $U$ ) and hopping $4 t$ (in units of $U$ ), as obtained from Eq. (8). Here, we set $C=0.2 U$, and $p / q=1 / 1(1 / 2)$ is shown as solid red (dashed black), corresponding to zero (maximum) magnetic field. The AMI and MI (SF) phases grow (shrinks) when the magnetic field increases from zero, due to the localizing effects of the magnetic field on bosons.

sions. First, we discussed the single-particle Hofstadter problem, and showed that the presence of a checkerboard superlattice gives rise to a magnetic flux-independent energy gap in the excitation spectrum. Then, we considered the manyparticle problem, and derived analytical mean-field expressions for the SF-MI and SF-AMI phase transition boundaries. We showed that the size of incompressible insulator phases grow when the magnetic field increases from zero, due to the localizing effect of the magnetic field on bosons. In addition, since the phase boundaries are functions of the minimal single-particle excitation energies, they have an intriguing dependence on the magnetic flux.

We also showed that the phase diagram of the BoseHubbard model on a checkerboard superlattice is in many ways similar to that of the extended Bose-Hubbard model. In particular, the compressible phase in the former model is more like a SS, where the SF and AMI orders coexist even for arbitrarily small $C$. However, since the checkerboard superlattice breaks the translational invariance of the lattice, we call the compressible phase of this model a SF to distinguish it from a true SS for which the translational invariance is broken spontaneously. For completeness, we discussed the effects of magnetic field on the the extended Bose-Hubbard model as well, and derived an analytical mean-field expression for the SF-MI and SF-CDW phase transition boundaries.
In this paper, we relied on the mean-field theory which is known to be sufficient in describing only the qualitative features of the phase diagram when there is no magnetic field. In particular, for the hardcore Bose-Hubbard model on a checkerboard superlattice, we have recently shown that the mean-field theory has a large quantitative discrepancy from the numerically exact Quantum Monte Carlo results especially in lower dimensions [16]. However, due to the infamous sign problem, such numerical calculations cannot be performed in the presence of a magnetic field, and therefore, it is not clear to us whether the mean-field theory is sufficient in this case or not. We hope that the intriguing structure of the phase transition boundaries on the minimal single-particle excitation energies of the Hofstadter butterfly, pedicted by the mean-field theory, could be observed in the experiments or verified via other exact means in the future.

We plan to extend this work at least in one important direction. There is some evidence that the ground-state phase diagram of the extended Bose-Hubbard model includes a SS phase, in dimensions higher than one $[22-24,26]$. The localizing effect of magnetic field on such a phase is yet to be studied, and it is not obvious whether SS region would grow or shrink when the magnetic field increases from zero. Although numerical calculations based on the Gutzwiller ansatz are expected to give gauge dependent results for the SS-SF phase boundary because of the mean-field nature of the ansatz, they would provide a good qualitative insight into this problem.

\section{ACKNOWLEDGMENTS}

The author thanks I. Hen for correspondence. This work is supported by the Marie Curie International Reintegration (Grant No. FP7-PEOPLE-IRG-2010-268239), Scientific and Technological Research Council of Turkey (Career Grant No. TÜBİTAK-3501-110T839), and the Turkish Academy of Sciences (TÜBA-GEBİP).

\section{Appendix A: Extended Bose-Hubbard model}

In many ways, the phase diagram of the Bose-Hubbard model on a checkerboard superlattice turned out to be similar to that of the extended Bose-Hubbard model. Therefore, in this appendix, we comment on the effects of uniform magnetic field on the insulating phases of the latter model. In contrast to our model where the translational invariance is broken due to checkerboard superlattice, the translational invariance is broken spontaneously in the extended model, leading to CDW modulations. The extended Bose-Hubbard Hamiltonian with the on-site $(U \geq 0)$ and nearest-neighbor $(V \geq 0)$ bosonboson repulsions can be written as

$$
\begin{aligned}
H= & -t \sum_{\langle i j\rangle}\left(e^{i \theta_{i j}} a_{i}^{\dagger} a_{j}+H . c .\right)+\frac{U}{2} \sum_{i} \widehat{n}_{i}\left(\widehat{n}_{i}-1\right) \\
& +V \sum_{\langle i j\rangle} \widehat{n}_{i} \widehat{n}_{j}-\mu \sum_{i} \widehat{n}_{i} .
\end{aligned}
$$


Here, we again consider a two-dimensional square optical lattice. For $U>4 V \neq 0$, it is well-known that the ground state has two types of insulating phases [22-25]. The first one is the MI phase where, similar to the usual Bose-Hubbard model, the ground-state boson occupancy is the same for every lattice site, i.e. $\left\langle\widehat{n}_{i}\right\rangle=n_{0}$. The second one is the CDW phase which has crystalline order in the form of staggered boson occupancies, i.e. $\left\langle\widehat{n}_{i}\right\rangle=n_{A}$ and $\left\langle\widehat{n}_{j}\right\rangle=n_{B}$ for $i$ and $j$ nearest neighbors.

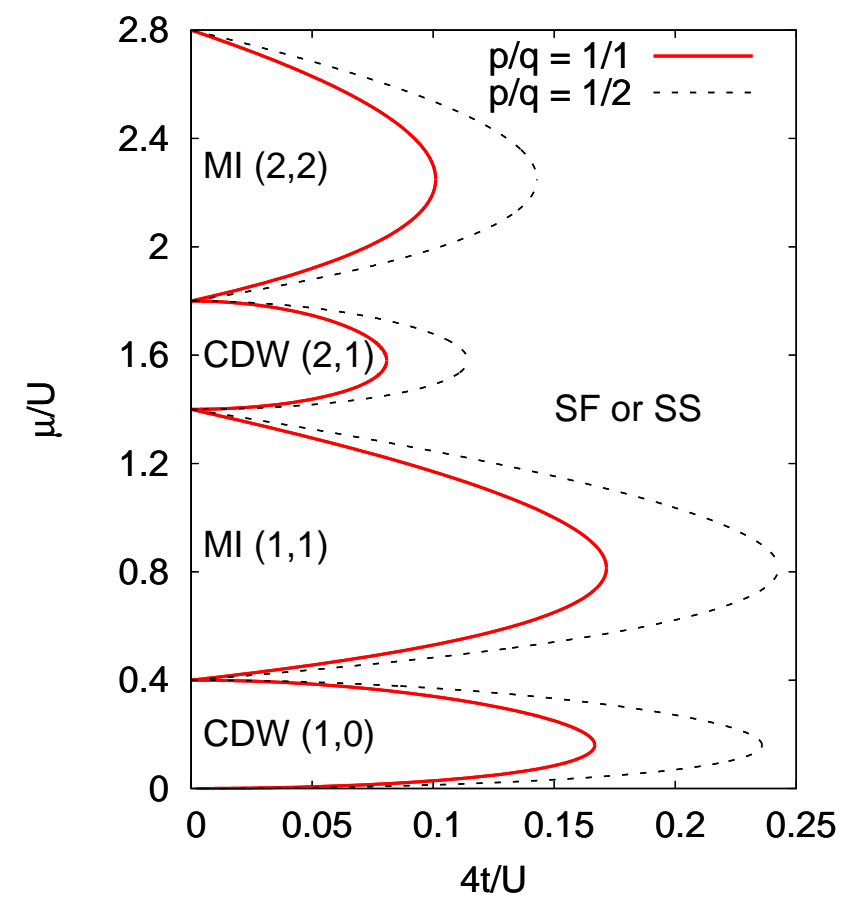

FIG. 5. (Color online) The ground-state phase diagram of the extended Bose-Hubbard model is shown as a function of the chemical potential $\mu$ (in units of $U$ ) and hopping $4 t$ (in units of $U$ ), as obtained from Eq. A2). Here, we set $V=0.1 U$, and $p / q=1 / 1(1 / 2)$ is shown as solid red (dashed black), corresponding to zero (maximum) magnetic field.

When $U>4 V$, in the atomic $t=0$ limit, the ground state alternates between the CDW and MI phases as a function of increasing $\mu$, where the chemical potential widths of CDW and MI lobes are $4 V$ and $U$, respectively. As $t$ increases, the range of $\mu$ about which the ground state is insulating decreases, and the MI and CDW phases disappear at a critical value of $t$, beyond which the system becomes compressible (SF or SS). On the other hand, the ground state has only CDW insulators when $U<4 V$. The chemical potential width of all CDW insulators is $U$, and the ground state is a CDW insulator with $\left(n_{0}, 0\right)$ fillings for $\left(n_{0}-1\right) U<\mu<n_{0} U$. As $t$ increases, the CDW phases disappear at a critical value of $t$, beyond which the system first becomes a SS then a SF at a much larger $t$ with a very large region of SS phase [26]. Within the mean-field decoupling theory, the phase transition boundaries are determined by

$$
\begin{aligned}
\frac{1}{\epsilon^{2}(\phi) t^{2}} & =\left[\frac{n_{A}+1}{U n_{A}+4 V n_{B}-\mu}-\frac{n_{A}}{U\left(n_{A}-1\right)+4 V n_{B}-\mu}\right] \\
\times & {\left[\frac{n_{B}+1}{U n_{B}+4 V n_{A}-\mu}-\frac{n_{B}}{U\left(n_{B}-1\right)+4 V n_{A}-\mu}\right], }
\end{aligned}
$$

which gives a quartic equation for $\mu$. Here, $\epsilon(\phi)=$ $\min _{\mathbf{k}} E_{0}(\phi) / t$ depends on the minimal single-particle excitation energies of the usual Hofstadter butterfly. Note that Eq. (A2) reduces to the known result for the nonmagnetic case [25] in the $\epsilon(\phi \rightarrow 0)=-4$ limit, and it reduces to the known magnetic result [5-7] when $n_{A}=n_{B}=n_{0}$ and $V=0$. Equation (A2) shows that the MI lobes are separated by $4 V$, but their shapes are independent of $V$ within the mean-field decoupling approximation; in particular, the critical points for the MI lobes are independent of $V$.

In Fig. 5, we set $V=0.1 U$ and show the ground-state phase diagram as a function of the chemical potential $\mu$ and hopping $4 t$ for $p / q=1 / 1$ (no magnetic flux) and for $p / q=1 / 2$ (maximum magnetic flux). As discussed above, the ground state alternates between the CDW and MI phases as a function of increasing $\mu$. The chemical potential widths of CDW and MI lobes are $0.4 U$ and $U$, respectively, but the size of the CDW (MI) lobes increase (decrease) as a function of increasing $V / U$ (not shown), since a nonzero $V$ is what allowed CDW states to form in the first place. In addition, the incompressible (compressible) CDW and MI (SF or SS) phases grow (shrinks) when the magnetic field increases from zero, due to the localizing effects of the magnetic field on bosons.
[1] I. Bloch, J. Dalibard, and W. Zwerger, Rev. Mod. Phys. 80, 885 (2008).

[2] M. Greiner, O. Mandel, T. Esslinger, T. W. Hänsch, and I. Bloch, Nature 415, 39 (2002).

[3] Y.-J. Lin, R. L. Compton, K. Jiménez-García, J. V. Porto, and I. B. Spielman, Nature 462, 628, (2009).

[4] Y.-J. Lin, R. L. Compton, A. R. Perry, W. D. Phillips, J. V. Porto, and I. B. Spielman, Phys. Rev. Lett. 102, 130401 (2009).

[5] M. Niemeyer, J. K. Freericks, and H. Monien, Phys. Rev. B, 60, 2357 (1999).

[6] M. Ö. Oktel, M. Nita, and B. Tanatar, Phys. Rev. B 75, 045133 (2007).
[7] R. O. Umucalılar and M. Ö. Oktel, Phys. Rev. A 76, 055601 (2007).

[8] D. S. Goldbaum and E. J. Mueller, Phys. Rev. A 79, 021602(R) (2009).

[9] S. Sinha and K. Sengupta, e-print arXiv:1003.0258 (2010).

[10] T. Duric and D. K. K. Lee, Phys. Rev. B 81, 014520 (2010).

[11] S. Powell, R. Barnett, R. Sensarma, and S. Das Sarma, Phys. Rev. Lett. 104, 255303 (2010).

[12] O. Tieleman, A. Lazarides, and C. Morais Smith, Phys. Rev. A 83, 013627 (2011).

[13] M. Aizenman, E. H. Lieb, R. Seiringer, J. P. Solovej, and J. Yngvason, Phys. Rev. A 70, 023612 (2004). 
[14] I. Hen and M. Rigol, Phys. Rev. B 80, 134508 (2009).

[15] V. G. Rousseau, D. P. Arovas, M. Rigol, F. Hébert, G. G. Batrouni, and R. T. Scalettar, Phys. Rev. B 73, 174516 (2006).

[16] I. Hen, M. Iskin, and M. Rigol, Phys. Rev. B 81, 064503 (2010).

[17] P. Buonsante and A. Vezzani, Phys. Rev. A 70, 033608 (2004).

[18] B.-L. Chen, S.-P. Kou, Y. Zhang, and S. Chen, Phys. Rev. A. 81, 053608 (2010).

[19] J. Sebby-Strabley, M. Anderlini, P. S. Jessen, and J. V. Porto, Phys. Rev. A 73, 033605 (2006).

[20] D. R. Hofstadter, Phys. Rev. B 14, 2239 (1976).

[21] This is in contrast to the usual Bose-Hubbard model where the first nontrivial hopping dependence of the phase transition boundary arises from the minimal eigenvalue of the $\mathbf{- t}$ matrix, such that $-\sum_{j} t_{i j} f_{j}=\epsilon(\phi) t f_{i}[5]$.

[22] C. Bruder, Rosario Fazio, and Gerd Schön, Phys. Rev. B 47, 342 (1993).

[23] Parhat Niyaz, R. T. Scalettar, C. Y. Fong, and G. G. Batrouni, Phys. Rev. B 50, 362 (1994).

[24] D. L. Kovrizhin, G. Venketeswara Pai, and S. Sinha, Europhys. Lett. 72, 162 (2005).

[25] M. Iskin and J. K. Freericks, Phys. Rev. A 80, 063610 (2009).

[26] M. Iskin, Phys. Rev. A 83, 051606(R) (2011). 